Preprint typeset in JHEP style - PAPER VERSION

SAGA-HE-253

KEK-TH-1321

\title{
Dominance of a single topological sector in gauge theory on non-commutative geometry
}

\author{
Hajime Aoki, $^{a}$ Jun Nishimura ${ }^{b, c}$ and Yoshiaki Susaki ${ }^{b}$ \\ ${ }^{a}$ Department of Physics, Saga University, Saga 840-8502, Japan \\ ${ }^{b}$ KEK Theory Center, High Energy Accelerator Research Organization, \\ Tsukuba, Ibaraki, 305-0801, Japan \\ ${ }^{c}$ Department of Particle and Nuclear Physics, \\ Graduate University for Advanced Studies (SOKENDAI), \\ Tsukuba, Ibaraki 305-0801, Japan \\ haoki@cc.saga-u.ac.jp, jnishi@post.kek.jp, susaki@post.kek.jp
}

\begin{abstract}
We demonstrate a striking effect of non-commutative (NC) geometry on topological properties of gauge theory by Monte Carlo simulations. We study 2d U(1) NC gauge theory for various boundary conditions using a new finite-matrix formulation proposed recently. We find that a single topological sector dictated by the boundary condition dominates in the continuum limit. This is in sharp contrast to the results in commutative space-time based on lattice gauge theory, where all topological sectors appear with certain weights in the continuum limit. We discuss possible implications of this effect in the context of string theory compactifications and in field theory contexts.
\end{abstract}

KeYwords: Non-Commutative Geometry, Nonperturbative Effects. 


\section{Contents}

1. Introduction 1

2. Brief review of the finite-matrix formulation 2

$\begin{array}{lll}\text { 3. } & \text { Monte Carlo results } & 6\end{array}$

4. Summary and discussions 11

\section{Introduction}

In matrix models of superstring theories [1,2], space and possibly also time are described by matrices, which are non-commutative from an outset. This matches well with the idea of non-commutative (NC) geometry [3, 4], which has been discussed for many years as possible effects of quantum gravity [5]. The connection became more concrete since field theories on a NC geometry were shown to appear naturally from matrix models $[6,7]$ and from string theories $[8]^{1}$. Dynamical properties of such NC field theories are therefore expected to play an important role in understanding fundamental nature of our real world.

In this paper, we demonstrate a striking effect of NC geometry on topological properties of gauge theory. We perform Monte Carlo simulations of $2 \mathrm{~d} U(1)$ gauge theory with various boundary conditions using a new finite-matrix formulation proposed recently [10]. The gauge field configurations are classified into topological sectors by the topological charge or the index of the Dirac operator. We find that a single topological sector dictated by the boundary condition dominates in the continuum limit. This is in sharp contrast to the results in commutative space-time based on lattice gauge theory [11, 12]. There, the distribution of the topological charge is gaussian with a finite extent. This means, in particular, that all topological sectors appear from a theory with a specific (e.g., periodic) boundary condition. Moreover, the width of the distribution diverges as the physical volume is increased, meaning that every topological sector appears with an equal weight in the thermodynamic limit.

We may interpret this striking difference as a kind of smoothing effects of NC geometry. Note that in both commutative and NC space-times, a regular configuration, which can be made smooth by an appropriate gauge transformation, has a specific topological charge fixed by the boundary condition. In commutative space-time, one can construct non-regular configurations without increasing the action considerably. This makes it possible to obtain a configuration in different topological sectors rather easily. In NC space-time, on the

\footnotetext{
${ }^{1}$ Recently the idea of emergent gravity is studied intensively [9] as another connection between quantum gravity and $\mathrm{NC}$ geometry.
} 
other hand, such non-regular configurations increase the action significantly since the starproduct contains all higher derivative terms. We confirm this point of view by calculating the average action for each topological sector. Indeed we observe that the average action is smaller in the specific topological sector than in the other sectors.

Thus, in NC gauge theory, one topological sector is singled out depending on the boundary condition. This may be important in the context of string theory compactifications, in which the topological properties in the extra dimensions determine, for instance, the number of generations. If the space-time in the extra dimensions are actually non-commutative (See refs. [13, 14] for discussions on models, in which extra dimensions appear as a fuzzy sphere.), our results suggest a mechanism for singling out a particular number of generations etc.. We also discuss possible implications on problems related to topological aspects of field theory such as the baryon number asymmetry and the strong CP problem.

The rest of this paper is organized as follows. In section 2 we briefly review the finitematrix formulation of gauge theories on a NC torus, which has been generalized recently to allow for twisted boundary conditions. In section 3 we present our Monte Carlo results for the distribution of topological sectors. Section 4 is devoted to a summary and discussions.

\section{Brief review of the finite-matrix formulation}

In this section we briefly review the finite-matrix formulation of gauge theories on a NC torus with twisted boundary conditions [10]. This is a generalization of the previous formulation for periodic boundary conditions [15]. The crucial point was to characterize the configuration space algebraically. As a simple example, we consider $2 \mathrm{~d} \mathrm{U}(p) \mathrm{NC}$ gauge theory with twisted boundary conditions, which correspond to introducing a constant background flux specified by the integer $q$. (We use the notations in ref. [10] except for switching the sign of the integer $q$, and hence the sign of $\tilde{q}$ defined in (2.5), to make some important formulae in this paper look nicer.)

For a gauge-singlet field, the boundary conditions reduce to the periodic ones, and the configuration space is given by the representation space of the coordinate operators $\hat{Z}_{\mu}=e^{2 \pi i \hat{x}_{\mu} / L}$ and the shift operators $\hat{\Gamma}_{\mu}=e^{\epsilon \hat{\partial}_{\mu}}$, which satisfy the algebra

$$
\begin{aligned}
\hat{Z}_{\mu} \hat{Z}_{\nu} & =e^{-2 \pi i \Theta_{\mu \nu}} \hat{Z}_{\nu} \hat{Z}_{\mu} \\
\hat{\Gamma}_{\mu} \hat{Z}_{\nu} \hat{\Gamma}_{\mu}^{\dagger} & =e^{\frac{2 \pi i}{N} \delta_{\mu \nu}} \hat{Z}_{\nu} \\
\hat{\Gamma}_{\mu} \hat{\Gamma}_{\nu} & =e^{-i \epsilon^{2} c_{\mu \nu}} \hat{\Gamma}_{\nu} \hat{\Gamma}_{\mu}
\end{aligned}
$$

Here $\Theta_{\mu \nu}$ and $c_{\mu \nu}$ represent non-commutativity, and $\epsilon$ serves as the lattice spacing with

$$
L=\epsilon N
$$

being the size of the torus.

For an adjoint field such as the gauge field, the theory with twisted boundary conditions can be mapped through the so-called Morita equivalence to a $\mathrm{U}\left(p_{0}\right) \mathrm{NC}$ gauge theory with periodic boundary conditions on a dual torus, where $p_{0}$ is the greatest common divisor of 
$p$ and $q$. For later convenience, let us introduce co-prime integers $\tilde{p}$ and $\tilde{q}$ by

$$
p=p_{0} \tilde{p}, q=p_{0} \tilde{q}
$$

and another set of integers $a$ and $b$ by the Diophantine equation

$$
a \tilde{p}-b \tilde{q}=1
$$

The covariant derivative operator $\hat{D}_{\mu}^{(0)}$ for the constant-curvature background field on the original torus is mapped to the derivative operator $\hat{\partial}_{\mu}^{\prime}=\hat{D}_{\mu}^{(0)}$ on the dual torus. Denoting the coordinate operators and the shift operators on the dual torus by $\hat{Z}_{\mu}^{\prime}$ and $\hat{\Gamma}_{\mu}^{\prime} \equiv e^{\epsilon \hat{\partial}_{\mu}^{\prime}}=$ $e^{\epsilon \hat{D}_{\mu}^{(0)}}$, we obtain the algebra

$$
\begin{aligned}
\hat{Z}_{\mu}^{\prime} \hat{Z}_{\nu}^{\prime} & =\mathrm{e}^{-2 \pi i \Theta_{\mu \nu}^{\prime}} \hat{Z}_{\nu}^{\prime} \hat{Z}_{\mu}^{\prime}, \\
\hat{\Gamma}_{\mu}^{\prime} \hat{Z}_{\nu}^{\prime} \hat{\Gamma}_{\mu}^{\prime \dagger} & =e^{\frac{2 \pi i}{n} \delta_{\mu \nu}} \hat{Z}_{\nu}^{\prime}, \\
\hat{\Gamma}_{\mu}^{\prime} \hat{\Gamma}_{\nu}^{\prime} & =e^{-i \epsilon^{2}\left(c_{\mu \nu}+f_{\mu \nu}\right)} \hat{\Gamma}_{\nu}^{\prime} \hat{\Gamma}_{\mu}^{\prime},
\end{aligned}
$$

where $\Theta_{\mu \nu}^{\prime}$ represents the non-commutativity tensor of the dual torus and $f_{\mu \nu}$ is the constant background flux specified by the integer $q$ as

$$
f_{12}=\frac{-2 \pi q}{L^{2}(p+\Theta q)}
$$

The non-commutativity tensors are written as $\Theta_{\mu \nu}=\Theta \varepsilon_{\mu \nu}$ and $\Theta_{\mu \nu}^{\prime}=\Theta^{\prime} \varepsilon_{\mu \nu}$ for the original and dual tori, respectively, where $\varepsilon_{\mu \nu}$ is the anti-symmetric tensor with $\varepsilon_{12}=1$. Then, the NC parameter $\Theta^{\prime}$ and the size

$$
L^{\prime}=\epsilon n
$$

of the dual torus are determined by those of the original torus as

$$
\begin{aligned}
\Theta^{\prime} & =\frac{a \Theta+b}{\tilde{p}+\tilde{q} \Theta}, \\
L^{\prime} & =L(\tilde{p}+\Theta \tilde{q}) .
\end{aligned}
$$

The configuration space of an adjoint field is given by the representation space of the operators $\hat{Z}_{\mu}^{\prime}$ and $\hat{\Gamma}_{\mu}^{\prime}$ satisfying the algebra (2.7)-(2.9).

From the above construction of the singlet and adjoint fields, it follows that the configuration space of a fundamental field is given by the representation space of the operators $\hat{Z}_{\mu}^{\prime}$ and $\hat{\Gamma}_{\mu}^{\prime}$ acting from the left, and the operators $\hat{Z}_{\mu}$ and $\hat{\Gamma}_{\mu}$ acting from the right.

Once the configuration space is characterized algebraically as above, we can represent the configurations by finite matrices by finding appropriate representations of the coordinate and shift operators. Since the operators $\hat{Z}_{\mu}$ and $\hat{\Gamma}_{\mu}$ are defined on the original torus, which is discretized into an $N \times N$ lattice, it is natural to represent them by $N \times N$ matri$\mathrm{ces}^{2}$ from the counting of degrees of freedom. Then the algebra (2.1)-(2.3) can be satisfied

\footnotetext{
${ }^{2}$ Their explicit form is given in eq. (5.6) of ref. [10].
} 
if the NC parameters are chosen as

$$
\begin{aligned}
\Theta & =\frac{2 r}{N}, \\
\epsilon^{2} c_{12} & =-2 \pi \frac{s}{N},
\end{aligned}
$$

where the integers $r$ and $s$ satisfy the Diophantine equation

$$
2 r s-k N=-1
$$

for some integer $k$. Similarly, since the operators $\hat{Z}_{\mu}^{\prime}$ and $\hat{\Gamma}_{\mu}^{\prime}$ are defined on the dual torus, which is discretized into an $n \times n$ lattice and endowed with the $\mathrm{U}\left(p_{0}\right)$ gauge group, it is natural to represent these operators by $n p_{0} \times n p_{0}$ matrices as

$$
\begin{aligned}
& \hat{Z}_{\mu}^{\prime}=Z_{\mu}^{(n)} \otimes \mathbb{1}_{p_{0}}, \\
& \hat{\Gamma}_{\mu}^{\prime}=\Gamma_{\mu}^{(n)} \otimes \mathbb{1}_{p_{0}} .
\end{aligned}
$$

The $n \times n$ matrices $^{3} Z_{\mu}^{(n)}$ and $\Gamma_{\mu}^{(n)}$ can satisfy the same algebra as (2.7)-(2.9) if we choose the NC parameters as

$$
\begin{aligned}
\Theta^{\prime} & =\frac{j}{n}, \\
\epsilon^{2}\left(c_{12}+f_{12}\right) & =2 \pi \frac{m}{n},
\end{aligned}
$$

where the integers $j$ and $m$ satisfy the Diophantine equation

$$
m j+n k^{\prime}=1
$$

for some integer $k^{\prime}$. The integers $m$ and $n$ of the dual torus are determined by those of the original torus, $N, r, s, k$, with the input of the integers $p$ and $q$ as

$$
m=-s \tilde{p}-k \tilde{q}, \quad n=N \tilde{p}+2 r \tilde{q} .
$$

The other integers $j$ and $k^{\prime}$ of the dual torus can be determined by eq. (2.21).

The action for the gauge field is given by the twisted Eguchi-Kawai model $[16,17]$

$$
S_{\mathrm{TEK}}=-n \beta^{\prime} \sum_{\mu \neq \nu} \mathcal{Z}_{\nu \mu}^{\prime} \operatorname{tr}\left(V_{\mu} V_{\nu} V_{\mu}^{\dagger} V_{\nu}^{\dagger}\right)+2 \beta^{\prime} n^{2} p_{0}
$$

where

$$
V_{\mu}=e^{\epsilon \hat{D}_{\mu}}
$$

are $\mathrm{U}\left(n p_{0}\right)$ matrices with $\hat{D}_{\mu}$ being the covariant derivative operator for the full gauge field including both the background and fluctuations. The $Z_{n}$ factor $\mathcal{Z}^{\prime}{ }_{\nu \mu}=\left(\mathcal{Z}^{\prime}{ }_{\mu \nu}\right)^{*}$ represents the twist, which is given by

$$
\mathcal{Z}^{\prime}{ }_{12}=\exp \left(-2 \pi i \frac{m}{n}\right)
$$

\footnotetext{
${ }^{3}$ Their explicit form is given in eq. (5.15) of ref. [10].
} 
The coefficient $\beta^{\prime}$ can be interpreted as the lattice coupling constant of the dual theory, which is related to that of the original theory $\beta$ as

$$
\beta=\frac{1}{\tilde{p}}\left(\frac{n}{N}\right)^{2} \beta^{\prime}
$$

The action (2.23) takes the minimum value and vanishes when $V_{\mu}=\hat{\Gamma}_{\mu}^{\prime} \equiv e^{\epsilon \hat{D}_{\mu}^{(0)}}$, which corresponds to the background gauge field. One can also show that the action (2.23) has the correct continuum limit classically.

Actions for fundamental matters can be given by using the covariant forward and backward difference operators $\nabla_{\mu}, \nabla_{\mu}^{*}$ defined by

$$
\begin{aligned}
\nabla_{\mu} \Psi & =\frac{1}{\epsilon}\left(V_{\mu} \Psi \hat{\Gamma}_{\mu}^{\dagger}-\Psi\right) \\
\nabla_{\mu}^{*} \Psi & =\frac{1}{\epsilon}\left(\Psi-V_{\mu}^{\dagger} \Psi \hat{\Gamma}_{\mu}\right) .
\end{aligned}
$$

Here $V_{\mu}$ is the $\mathrm{U}\left(n p_{0}\right)$ matrix introduced by $(2.24)$, and $\hat{\Gamma}_{\mu}=\mathrm{e}^{\epsilon \hat{\partial}_{\mu}}$ is the shift operator represented by an $N \times N$ matrix satisfying (2.3) and (2.15). The fundamental matter field $\Psi$ is represented by $n p_{0} \times N$ rectangular matrices. One can define an overlap Dirac operator [18] $\mathrm{as}^{4}$

$$
D=\frac{1}{\epsilon}\left(1-\gamma_{5} \hat{\gamma}_{5}\right)
$$

where $\gamma_{5}$ is the ordinary chirality operator and $\hat{\gamma}_{5}$ is the modified one defined by

$$
\begin{aligned}
& \hat{\gamma}_{5}=\frac{H}{\sqrt{H^{2}}}, \\
& H=\gamma_{5}\left(1-\epsilon D_{\mathrm{W}}\right)
\end{aligned}
$$

in terms of the Wilson-Dirac operator

$$
D_{\mathrm{W}}=\frac{1}{2} \sum_{\mu=1}^{2}\left\{\gamma_{\mu}\left(\nabla_{\mu}^{*}+\nabla_{\mu}\right)-\epsilon \nabla_{\mu}^{*} \nabla_{\mu}\right\} .
$$

The Dirac operator (2.28) satisfies the Ginsparg-Wilson relation [22]

$$
\gamma_{5} D+D \hat{\gamma}_{5}=0
$$

which guarantees the exact chiral symmetry [23]. Thanks to the index theorem [24], one can classify gauge configurations into topological sectors using the index of $D$ given by

$$
\nu=\frac{1}{2} \mathcal{T} r\left(\gamma_{5}+\hat{\gamma}_{5}\right)=\frac{1}{2} \mathcal{T} r \hat{\gamma}_{5},
$$

where the trace $\mathcal{T r}$ is taken in the configuration space of the matter field.

\footnotetext{
${ }^{4}$ The overlap Dirac operator was introduced on a periodic NC torus in ref. [19], and the correct form of the axial anomaly has been reproduced in the continuum limit [20]. A prescription to define an analog of the overlap Dirac operator and its index (2.33) on general NC manifolds including the fuzzy sphere has been proposed in ref. [21].
} 
The topological charge can also be defined as ${ }^{5}$

$$
Q=\frac{1}{4 \pi i} N \sum_{\mu \neq \nu} \epsilon_{\mu \nu} \mathcal{Z}_{\nu \mu} \operatorname{tr}\left(V_{\mu} V_{\nu} V_{\mu}^{\dagger} V_{\nu}^{\dagger}\right)
$$

with the $Z_{N}$ factor $\mathcal{Z}_{\nu \mu}=\left(\mathcal{Z}_{\mu \nu}\right)^{*}$ given by

$$
\mathcal{Z}_{12}=\exp \left(2 \pi i \frac{s}{N}\right)
$$

For $V_{\mu}=\hat{\Gamma}_{\mu}^{\prime}$, which gives the minimum of the gauge action (2.23), the topological charge becomes $Q=q$ as expected. One can also show that $Q$ has the correct continuum limit classically. Note, however, that $Q$ does not take integer values for generic configurations unlike the index $\nu$.

\section{Monte Carlo results}

In this section we present our results for the probability distribution of the index (2.33) and the topological charge (2.34) obtained by simulating the model (2.23). Details of the simulation are described in ref. [27].

First we comment on the parameters we have chosen. For simplicity, we consider U(1) gauge group $p=1$, which also implies $p_{0}=1$ and hence $\tilde{p}=1, \tilde{q}=q$. As for the integers $r$ and $k$ appearing in eq. (2.16), we choose $r=-1, k=-1$ (and hence $s=\frac{N+1}{2}$ ) following essentially the choice in the previous works $[27,25,28,10]$. This implies, in particular, that the NC parameter (2.14) is given by $\Theta=-2 / N$, where $N$ represents the size of the torus (2.4) in units of the lattice spacing. Note that the size $n$ of the matrices $V_{\mu}$ and the integer $m$, which labels the twist (2.25) in the gauge action (2.23), are given by $n=N-2 q$ and $m=-(n+1) / 2$, respectively, due to $(2.22)$.

We perform Monte Carlo simulations for various values of $q$. We measure the index $\nu$ and the topological charge $Q$ for each configuration generated by Monte Carlo simulation, and obtain the probability distribution $P(\nu)$ and $P(Q)$.

Let us present our results for the index $\nu$ given by (2.33). In fig. 1 we plot the distribution of the index for $q=-2,-1,0,1,2$. (The results for $q=0$ are already given in ref. [28].) On the left we show how the probability distribution of $\nu$ changes as we increase $\beta^{\prime}$ for $n=15$. (We assume the normalization $\sum_{\nu} P(\nu)=1$.) We find that the probability for $\nu \neq q$ decreases rapidly, and the probability for $\nu=q$ approaches unity. On the right we plot the probability distribution $P(\nu)$ for various $n$ at $\beta^{\prime}=0.55$. (Note that the value of $\beta^{\prime}$ we have chosen lies in the region above the critical point $\beta^{\prime}=\beta_{\text {cr }} \equiv 1 / 2$ of the Gross-Witten phase transition [29], which is relevant for the continuum limit.) We find that the distribution approaches the Kronecker delta $\delta_{\nu, q}$ not only for increasing $\beta^{\prime}$ but also for increasing $n$.

\footnotetext{
${ }^{5}$ This reduces to the one in ref. [25] for $q=0$. An analogous definition was also used in ref. [26]. Their topological charge $Q_{\mathrm{GS}}$ is related to ours by $Q=-Q_{\mathrm{GS}} / 2 \pi$.
} 

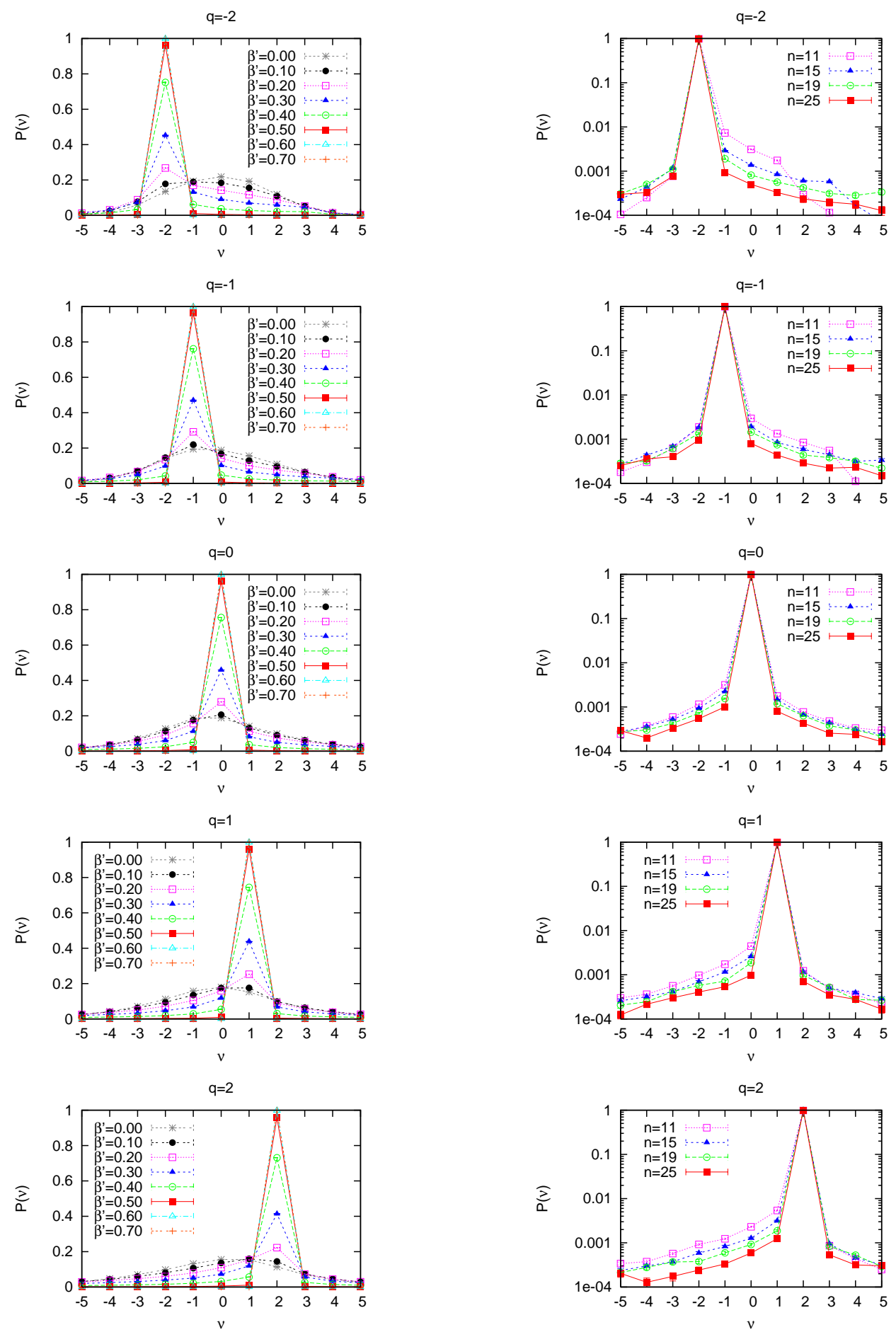

Figure 1: The probability distribution of the index $\nu$ is plotted for various $\beta^{\prime}$ at $n=15$ (left) and for various $n$ at $\beta^{\prime}=0.55$ (right). From the top to the bottom, we present the results for $q=-2,-1,0,1,2$. In the plots on the right, the probability is plotted in the log scale to make the distribution at $\nu \neq q$ visible. 

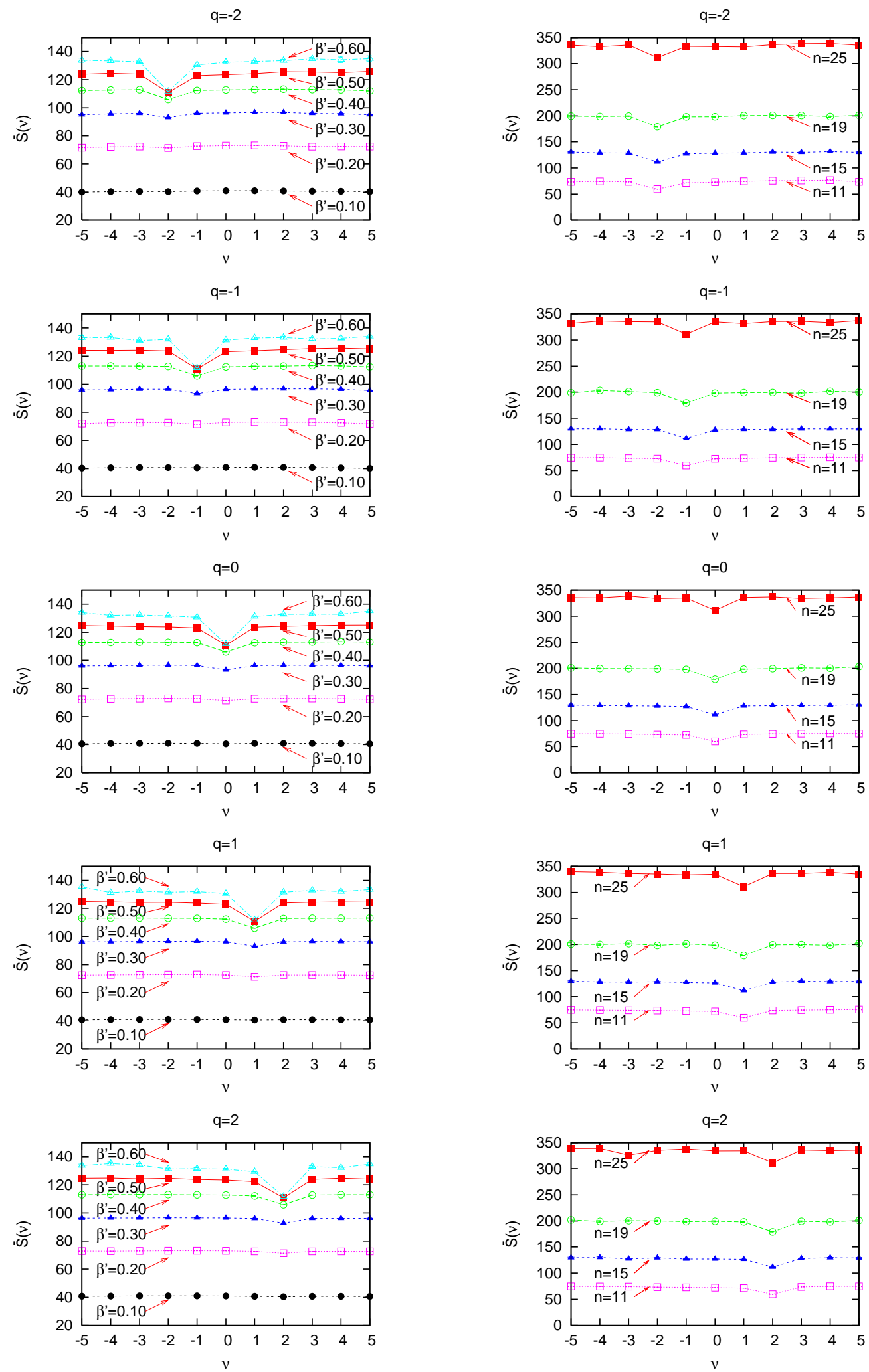

Figure 2: The average value of the action is plotted against the index $\nu$ for various $\beta^{\prime}$ at $n=15$ (left) and for various $n$ at $\beta^{\prime}=0.55$ (right). From the top to the bottom, we present the results for $q=-2,-1,0,1,2$. 

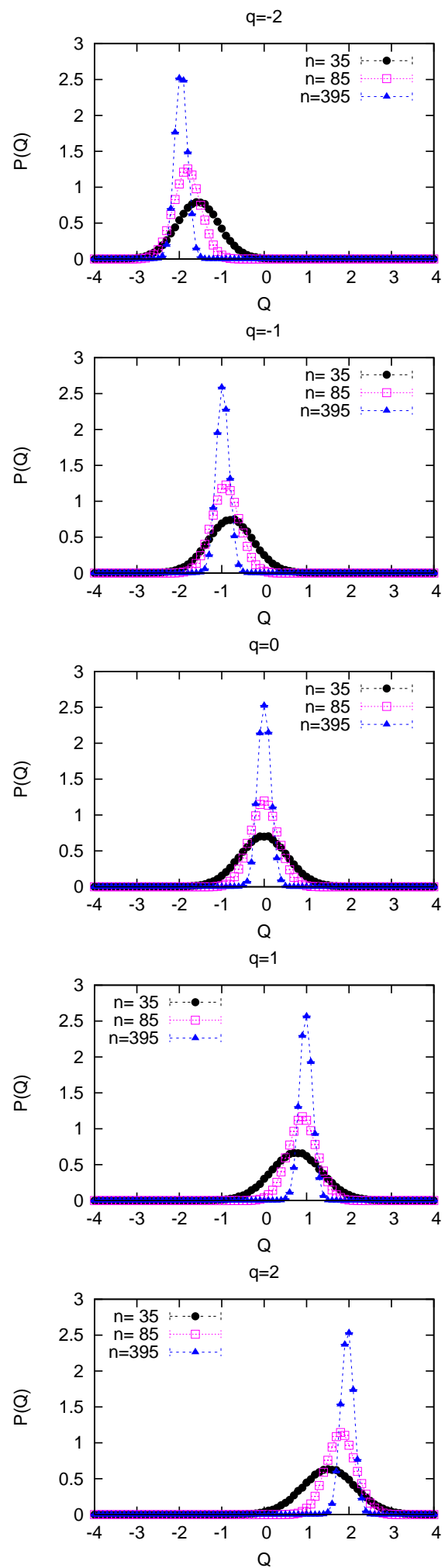

Figure 3: The probability distribution of the topological charge $Q$ is plotted for $q=-2,-1,0,1,2$ with $n=35,85,395$ and fixed $n / \beta^{\prime}=32$. 

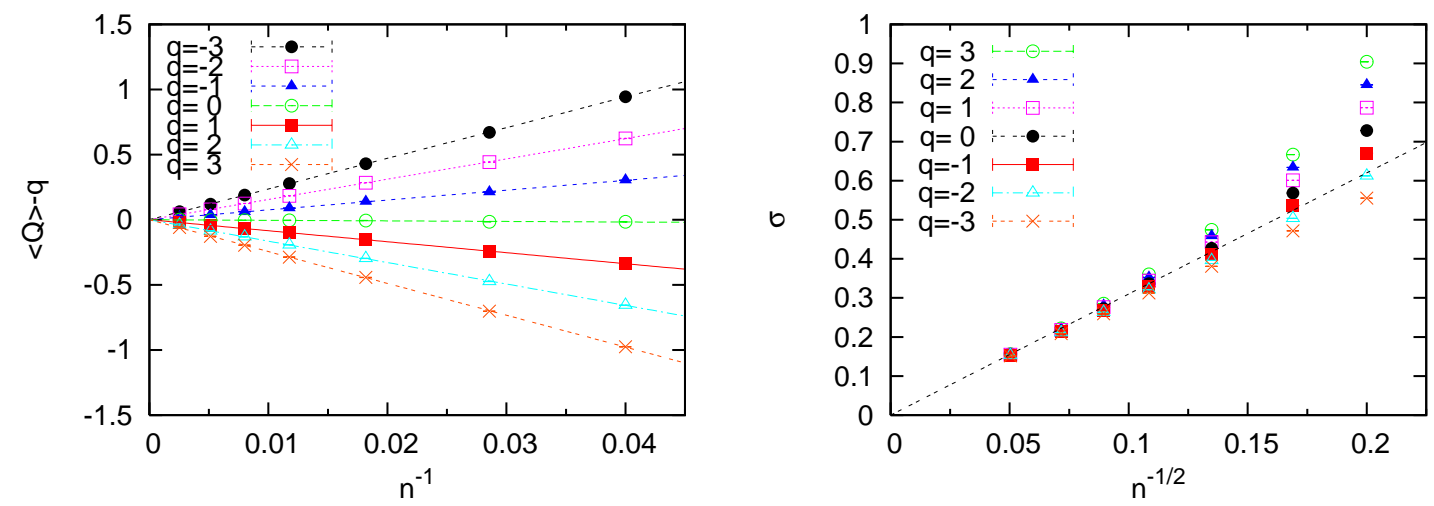

Figure 4: (Left) The discrepancy of the vacuum expectation value of the topological charge $Q$ from $q$ is plotted against $1 / n$. (Right) The width of the probability distribution of $Q$ is plotted against $1 / \sqrt{n}$.

In order to take the continuum limit, we have to send $n$ and $\beta^{\prime}$ to infinity simultaneously fixing the ratio $n / \beta^{\prime}[27] .{ }^{6}$ It is clear from the above results that the distribution $P(\nu)$ approaches $\delta_{\nu, q}$ very rapidly in that limit. This conclusion provides a physical interpretation to the results of the instanton calculus in the continuum theory [30], where the partition function has been written as a sum over all the instanton configurations with a certain constraint related to the magnetic flux $q$.

In the commutative case, analytical and numerical studies show that the distribution of the topological charge is gaussian with a finite width, and the width diverges in the infinite-volume limit $[11,12]$. Thus the situation in the NC case differs drastically from the commutative case. This can be interpreted as a kind of smoothing effects of NC geometry due to the existence of all higher derivative terms in the star product as we discussed in the Introduction. To substantiate this argument, we plot in fig. 2 the average value of the action $\bar{S}(\nu)$ in each topological sector. We find that the result is almost independent of $\nu$ except for $\nu=q$, where we indeed observe a dip.

Next we present our results for the topological charge $Q$ defined by (2.34). In fig. 3 the probability distribution of $Q$ is plotted for $q=-2,-1,0,1,2$, showing how it changes as we take the continuum limit, ${ }^{7}$ i.e., sending $n$ and $\beta^{\prime}$ to infinity with fixed $n / \beta^{\prime}$. Here we fix it to $n / \beta^{\prime}=32$ following ref. [27]. (We assume the normalization $\int d Q P(Q)=1$.) We find that the distribution is a gaussian peaked slightly away from the integer $q$. From fig. 4 , however, we find in the continuum limit that the deviation $\langle Q\rangle-q$ vanishes as $1 / n$, and the width of the distribution vanishes as $1 / \sqrt{n}$. Thus we have confirmed that the distribution of the topological charge becomes a delta function peaked at $q$. This is consistent with the

\footnotetext{
${ }^{6}$ The "lattice spacing" $\epsilon$ has to be sent to zero in such a way that the dimensionful coupling constant $g^{2}=\frac{1}{\epsilon^{2} \beta}$ is fixed. We also require the dimensionful non-commutativity parameter $\Theta L^{2} \sim N \epsilon^{2}$ to be finite. These two conditions imply fixing the ratio $N / \beta \sim n / \beta^{\prime}$ in the continuum limit. The size of the torus $L=N \epsilon$ diverges as $\sqrt{N} \sim \sqrt{n}$ in that limit.

${ }^{7}$ In ref. [31], growing of the peak is observed as one increases $\beta^{\prime}$ with fixed $n$ for $q=0$.
} 
index theorem $[32,33]$, which asserts that the index $\nu$ agrees with the topological charge $Q$ for configurations that survive in the continuum limit.

Let us recall that the definition of the topological charge we used is given by eq. (2.34), which may be viewed as a naive discretization of the corresponding expression in the continuum. In the commutative space, one can use the geometrical construction [34] to define an integer topological charge for any lattice configuration. It takes a particularly simple form in the $2 \mathrm{~d} \mathrm{U}(1)$ case, which is used in ref. [11, 12]. In $4 \mathrm{~d}$ theories, on the other hand, one usually uses a naively defined topological charge, but one can obtain a distribution peaked at integer values by using some techniques like cooling or renormalization. (See ref. [35], for instance.) Note that here we are able to obtain a distribution peaked at an integer value even with the naive definition of the topological charge without any techniques. This might also be due to smoothing effects of NC geometry. Let us also mention that the computational effort for calculating $Q$ is of order $\mathrm{O}\left(n^{3}\right)$, whereas that for calculating the index $\nu$ is of order $\mathrm{O}\left(n^{6}\right)$. Therefore, $Q$ might be of some use when one tries to extend the present work to higher dimensions.

\section{Summary and discussions}

In this paper, we have studied the probability distribution of the index $\nu$ and the topological charge $Q$ in the finite-matrix formulation of $2 \mathrm{~d} \mathrm{U}(1) \mathrm{NC}$ gauge theory with various boundary conditions. Our results suggest that a single topological sector, which is dictated by the boundary condition specified by the integer $q$, dominates in the continuum limit. ${ }^{8}$ This is in sharp contrast to the situation in the ordinary lattice gauge theory, where all the topological sectors appear from a single theory. This striking difference can be interpreted as a kind of smoothing effects of NC geometry.

Let us discuss possible implications of our results to the real world. Considering that our space-(time) naturally becomes non-commutative, for instance, in matrix model formulations $[1,2]$ of string theory, we may expect that NC geometry is realized in nature in various ways, which we discuss in what follows separately.

First we consider the possibility that NC geometry is realized in the extra dimensions $[13,14]$. There is a well-known scenario in string theory that the number of generations, for instance, is determined by the index in the extra dimensions. However, its distribution may have a non-vanishing width in general due to dynamical fluctuations of the gauge fields in the extra dimensions, which causes a serious problem in this scenario. If the extradimensional space is non-commutative, the index takes a specific value once the boundary condition is fixed somehow, even if the dynamical fluctuations are taken into account.

We can also consider that our $4 \mathrm{~d}$ space-time has certain non-commutativity. In this case, non-locality may cause some problems such as the violation of causality, renormal-

\footnotetext{
${ }^{8}$ In the literature, the possibility of summing over boundary conditions is sometimes discussed. In this case, the relative weight should be fixed by some underlying principle. For example, in the random matrix theory for QCD, the relative weight is fixed by the guiding principle that one should recover QCD [36]. Alternatively, one can introduce the Higgs sector in the NC gauge theory [37] (See [38] for a related work.). In this case, the winding number of the Higgs field plays the role of the twisted boundary condition, and all the topological sectors appear from a single theory.
} 
izability and the CPT theorem. It also induces the UV/IR mixing effects, which make it difficult to think of phenomenologically viable models. From the viewpoint of string theory, it is a big challenge to understand how one can get rid of all these problems arising from non-locality. (See ref. [39] for a different way to introduce NC geometry without inducing the UV/IR mixing effects.) Let us assume here that they are resolved somehow, and discuss what would be other consequences of the NC geometry. In particular, we speculate on how the new properties we found in the present paper may play roles in various problems related to topological aspects of gauge theories.

Let us consider the baryon number asymmetry of the universe. In the electroweak theory, the baryon number conservation is violated by the chiral anomaly through instanton effects. In order to generate net baryon number asymmetry, one usually introduces some bias caused, for instance, by a bubble wall sweeping over the universe during the electroweak phase transition. Our result suggests that NC geometry with a twisted boundary condition may provide a simple and direct source for the net asymmetry.

We also speculate that the strong CP problem may be solved by NC geometry. From the experimental bound on the electric dipole moment, the parameter $\bar{\theta}$ of the QCD vacuum (including the phase of the fermion mass) must be less than $10^{-9}$, which needs some explanation. If the space-time is given by a $\mathrm{NC}$ geometry, a single topological sector with a specific value of $Q$ dominates, and the $e^{i \bar{\theta} Q}$ simply factors out of the path integral. Hence the physics do not depend on the parameter $\bar{\theta}$. This explanation is reminiscent of the scenario [40] that the long-range interaction between instantons and anti-instantons washes out the local topological structure, which makes the topology of the gauge field determined only by the boundary condition.

We hope that some of the above speculations can be pursued further by investigating the relevance of matrix model formulations of string theory to our world, and by studying NC gauge theories in four dimensions by Monte Carlo simulations [41].

\section{Acknowledgments}

We thank Satoshi Iso, Hikaru Kawai and Hiroshi Yoneyama for valuable discussions.

\section{References}

[1] T. Banks, W. Fischler, S. H. Shenker and L. Susskind, M theory as a matrix model: A conjecture, Phys. Rev. D 55 (1997) 5112 [hep-th/9610043].

[2] N. Ishibashi, H. Kawai, Y. Kitazawa, and A. Tsuchiya, A large- $N$ reduced model as superstring, Nucl. Phys. B 498 (1997) 467 [hep-th/9612115].

[3] H.S. Snyder, Quantized space-time, Phys. Rev. 71 (1947) 38.

[4] A. Connes, Noncommutative geometry, Academic Press, 1990.

[5] S. Doplicher, K. Fredenhagen and J.E. Roberts, The quantum structure of spacetime at the Planck scale and quantum fields, Commun. Math. Phys. 172 (1995) 187 [hep-th/0303037].

[6] A. Connes, M. R. Douglas and A. Schwarz, Noncommutative geometry and matrix theory: Compactification on tori, J. High Energy Phys. 02 (1998) 003 [hep-th/9711162]. 
[7] H. Aoki, N. Ishibashi, S. Iso, H. Kawai, Y. Kitazawa and T. Tada, Noncommutative Yang-Mills in IIB matrix model, Nucl. Phys. B 565 (2000) 176 [hep-th/9908141].

[8] N. Seiberg and E. Witten, String theory and non-commutative geometry, J. High Energy Phys. 09 (1999) 032 [hep-th/9908142].

[9] H. Steinacker, Emergent gravity from noncommutative gauge theory, J. High Energy Phys. 12 (2007) 049 [arXiv:0708.2426]; R. Delgadillo-Blando, D. O'Connor and B. Ydri, Geometry in transition: a model of emergent geometry, Phys. Rev. Lett. 100 (2008) 201601 [arXiv:0712.3011]; H. Grosse, H. Steinacker and M. Wohlgenannt, Emergent gravity, matrix models and UV/IR mixing, J. High Energy Phys. 04 (2008) 023 [arXiv:0802.0973];

H. Steinacker, Emergent gravity and noncommutative branes from Yang-Mills matrix models, Nucl. Phys. B 810 (2009) 1 [arXiv:0806.2032]; H. S. Yang, Emergent spacetime and the origin of gravity, J. High Energy Phys. 05 (2009) 012 [arXiv:0809.4728].

[10] H. Aoki, J. Nishimura and Y. Susaki, Finite-matrix formulation of gauge theories on a non-commutative torus with twisted boundary conditions, J. High Energy Phys. 04 (2009) 055 [arXiv:0810.5234].

[11] A. S. Hassan, M. Imachi, N. Tsuzuki and H. Yoneyama, Character expansion, zeros of partition function and theta term in U(1) gauge theory, Prog. Theor. Phys. 94 (1995) 861 [hep-lat/9508011].

[12] C. R. Gattringer, I. Hip and C. B. Lang, Quantum fluctuations versus topology: A study in U(1) $)_{2}$ lattice gauge theory, Phys. Lett. B 409 (1997) 371 [hep-lat/9706010].

[13] P. Aschieri, J. Madore, P. Manousselis and G. Zoupanos, Dimensional reduction over fuzzy coset spaces, J. High Energy Phys. 04 (2004) 034 [hep-th/0310072]; P. Aschieri,

T. Grammatikopoulos, H. Steinacker and G. Zoupanos, Dynamical generation of fuzzy extra dimensions, dimensional reduction and symmetry breaking, J. High Energy Phys. 09 (2006) 026 [hep-th/0606021]; H. Steinacker and G. Zoupanos, Fermions on spontaneously generated spherical extra dimensions, J. High Energy Phys. 09 (2007) 017 [arXiv:0706.0398].

[14] H. Aoki, S. Iso, T. Maeda and K. Nagao, Dynamical generation of a nontrivial index on the fuzzy 2-sphere, Phys. Rev. D 71 (2005) 045017 [hep-th/0412052], Erratum : Phys. Rev. D 71 (2005) 069905.

[15] J. Ambjørn, Y.M. Makeenko, J. Nishimura and R.J. Szabo, Finite $N$ matrix models of noncommutative gauge theory, J. High Energy Phys. 11 (1999) 029 [hep-th/9911041]; Nonperturbative dynamics of noncommutative gauge theory, Phys. Lett. B 480 (2000) 399 [hep-th/0002158]; Lattice gauge fields and discrete noncommutative Yang-Mills theory, J. High Energy Phys. 05 (2000) 023 [hep-th/0004147].

[16] T. Eguchi and H. Kawai, Reduction of dynamical degrees of freedom in the large $N$ gauge theory, Phys. Rev. Lett. 48 (1982) 1063.

[17] A. González-Arroyo and M. Okawa, A twisted model for large $N$ lattice gauge theory, Phys. Lett. B 120 (1983) 174; The twisted Eguchi-Kawai model: A reduced model for large $N$ lattice gauge theory, Phys. Rev. D 27 (1983) 2397.

[18] H. Neuberger, Exactly massless quarks on the lattice, Phys. Lett. B 417 (1998) 141 [hep-lat/9707022]; Vector like gauge theories with almost massless fermions on the lattice, Phys. Rev. D 57 (1998) 5417 [hep-lat/9710089]; More about exactly massless quarks on the lattice, Phys. Lett. B 427 (1998) 353 [hep-lat/9801031]. 
[19] J. Nishimura and M. A. Vazquez-Mozo, Noncommutative chiral gauge theories on the lattice with manifest star-gauge invariance, J. High Energy Phys. 08 (2001) 033 [hep-th/0107110].

[20] S. Iso and K. Nagao, Chiral anomaly and Ginsparg-Wilson relation on the noncommutative torus, Prog. Theor. Phys. 109 (2003) 1017 [hep-th/0212284].

[21] H. Aoki, S. Iso and K. Nagao, Ginsparg-Wilson relation, topological invariants and finite noncommutative geometry, Phys. Rev. D 67 (2003) 085005 [hep-th/0209223].

[22] P. H. Ginsparg and K. G. Wilson, A remnant of chiral symmetry on the lattice, Phys. Rev. D 25 (1982) 2649.

[23] M. Lüscher, Exact chiral symmetry on the lattice and the Ginsparg-Wilson relation, Phys. Lett. B 428 (1998) 342 [hep-lat/9802011].

[24] P. Hasenfratz, V. Laliena and F. Niedermayer, The index theorem in QCD with a finite cut-off, Phys. Lett. B 427 (1998) 125 [hep-lat/9801021].

[25] H. Aoki, J. Nishimura and Y. Susaki, The index of the overlap Dirac operator on a discretized 2d non-commutative torus, J. High Energy Phys. 02 (2007) 033 [hep-th/0602078].

[26] L. Griguolo and D. Seminara, Classical solutions of the TEK model and noncommutative instantons in two dimensions, J. High Energy Phys. 03 (2004) 068 [hep-th/0311041].

[27] W. Bietenholz, F. Hofheinz and J. Nishimura, A non-perturbative study of gauge theory on a non-commutative plane, J. High Energy Phys. 09 (2002) 009 [hep-th/0203151].

[28] H. Aoki, J. Nishimura and Y. Susaki, Probability distribuion of the index in gauge theory on $2 d$ non-commutative geometry, J. High Energy Phys. 10 (2007) 024 [hep-th/0604093].

[29] D.J. Gross and E. Witten, Possible third order phase transition in the large $N$ lattice gauge theory, Phys. Rev. D 21 (1980) 446.

[30] L. D. Paniak and R. J. Szabo, Instanton expansion of noncommutative gauge theory in two dimensions, Commun. Math. Phys. 243 (2003) 343 [hep-th/0203166].

[31] W. Frisch, H. Markum and H. Grosse, Instantons in two-dimensional noncommutative U(1) gauge theory, PoS LAT2007 (2007) $31 \%$.

[32] M. F. Atiyah and I. M. Singer, The index of elliptic operators. 5, Annals Math. 93 (1971) 139.

[33] K. Y. Kim, B. H. Lee and H. S. Yang, Zero-modes and Atiyah-Singer index in noncommutative instantons, Phys. Rev. D 66 (2002) 025034 [hep-th/0205010].

[34] M. Lüscher, Topology of lattice gauge fields, Commun. Math. Phys. 85 (1982) 39.

[35] E. Vicari and H. Panagopoulos, Theta dependence of $S U(N)$ gauge theories in the presence of a topological term, Phys. Rev. 470 (2009) 93 [arXiv:0803.1593].

[36] E. V. Shuryak and J. J. M. Verbaarschot, Random matrix theory and spectral sum rules for the Dirac operator in QCD, Nucl. Phys. A 560 (1993) 306 [hep-th/9212088]; C. Lehner, M. Ohtani, J. J. M. Verbaarschot and T. Wettig, Topology and chiral random matrix theory at nonzero imaginary chemical potential, arXiv:0902.2640 [hep-th].

[37] H. Aoki, S. Iso and T. Maeda, Ginsparg-Wilson Dirac operator in the monopole backgrounds on the fuzzy 2-sphere, Phys. Rev. D 75 (2007) 085021 [hep-th/0610125]; H. Aoki, Y. Hirayama and S. Iso, Index theorem in spontaneously symmetry-broken gauge theories on fuzzy 2-sphere, Phys. Rev. D 78 (2008) 025028 [arXiv:0804.0568]. 
[38] H. Steinacker, Quantized gauge theory on the fuzzy sphere as random matrix model, Nucl. Phys. B 679 (2004) 66 [hep-th/0307075].

[39] A. P. Balachandran, T. R. Govindarajan, G. Mangano, A. Pinzul, B. A. Qureshi and S. Vaidya, Statistics and UV-IR mixing with twisted Poincare invariance, Phys. Rev. D 75 (2007) 045009 [hep-th/0608179].

[40] S. Samuel, Does the Yang-Mills theory solve the strong CP Problem by itself?, Mod. Phys. Lett. A 7 (1992) 2007.

[41] W. Bietenholz, J. Nishimura, Y. Susaki and J. Volkholz, A non-perturbative study of 4d U(1) non-commutative gauge theory: The fate of one-loop instability, J. High Energy Phys. 10 (2006) 042 [hep-th/0608072]. 